\title{
Contactin-2 associated protein autoimmune neurological conditions: detailed clinical phenotype and antibody quantification
}

\section{Varley JA, Binks S, Waters P and Irani SR}

250 Words

Contactin-2 association protein (CASPR2) antibodies are associated with neurological symptoms which are predominantly peripheral (neuromyotonia), central (limbic encephalitis) or combinations of both (Morvan's Syndrome). The precise nature of these phenotypes requires further study. Also, comparative utilities of different methods to detect CASPR2-antibody have not been presented. Therefore, we performed detailed clinical interviews, cognitive assessments and obtained serum from 20 patients with positive CASPR2-antibodies by cell based assay (CBA), and compared this gold-standard with a fluorescence immunoprecipitation assay (FIPA) which is intrinsically a higher-throughput and more quantitative assay.

17 of 20 patients were male (85\%) with a median age of 69 (range $26-81$ ). Symptom frequency during disease was as follows: seizures (75\%), neuropsychiatric $(70 \%$, sleep abnormality (50\%), nerve pain (40\%) autonomic dysfunction $(30 \%$,$) and neuromyotonia$ (30\%). By comparison to our previous cohort, only 4 patients (20\%) had associated cancer (2 prostate, 1 thyroid, 1 bowel cancer). Cognitive outcomes in recovery (median, range) showed a median Addenbrooke's Cognitive Examination (ACE-R) score of 93 (range 75-99). On follow up, common residual features included neuropathic pain, six patients (30\%) and fatigue, two patients (10\%). The FIPA was able to diagnose $11 / 16$ patients that were positive on CBA (Figure 1, sensitivity 69\%) and positive results showed a good correlation with CBAbased end-titres (Figure 2, Spearman r of 0.93 [95\% Cl 0.80 to 0.98$]$ ).

This work extends previous findings on the demographics and symptomatology of patients with CASPR2-antibodies, and further quantifies the cognitive outcome and longterm neuropathic pain, as well as an alternative method of quantifying CASPR2-antibody concentration. 
Figure 1. CASPR2 patients versus healthy controls

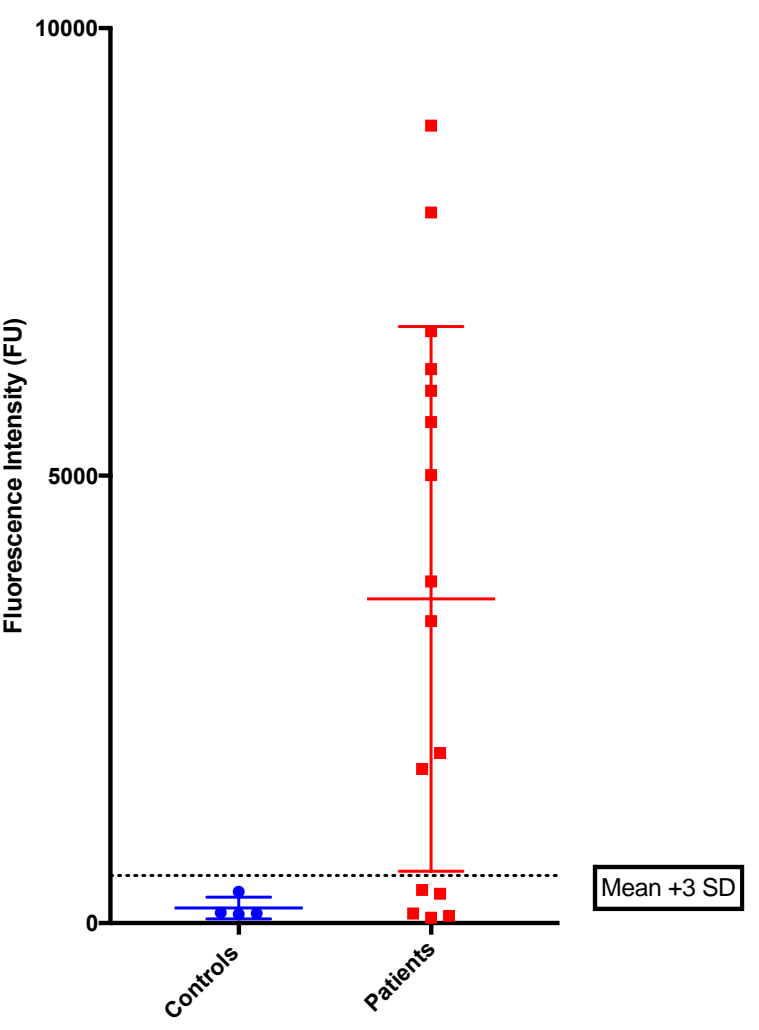

Figure 2. CASPR2 patient end titre titre vs FIPA FU

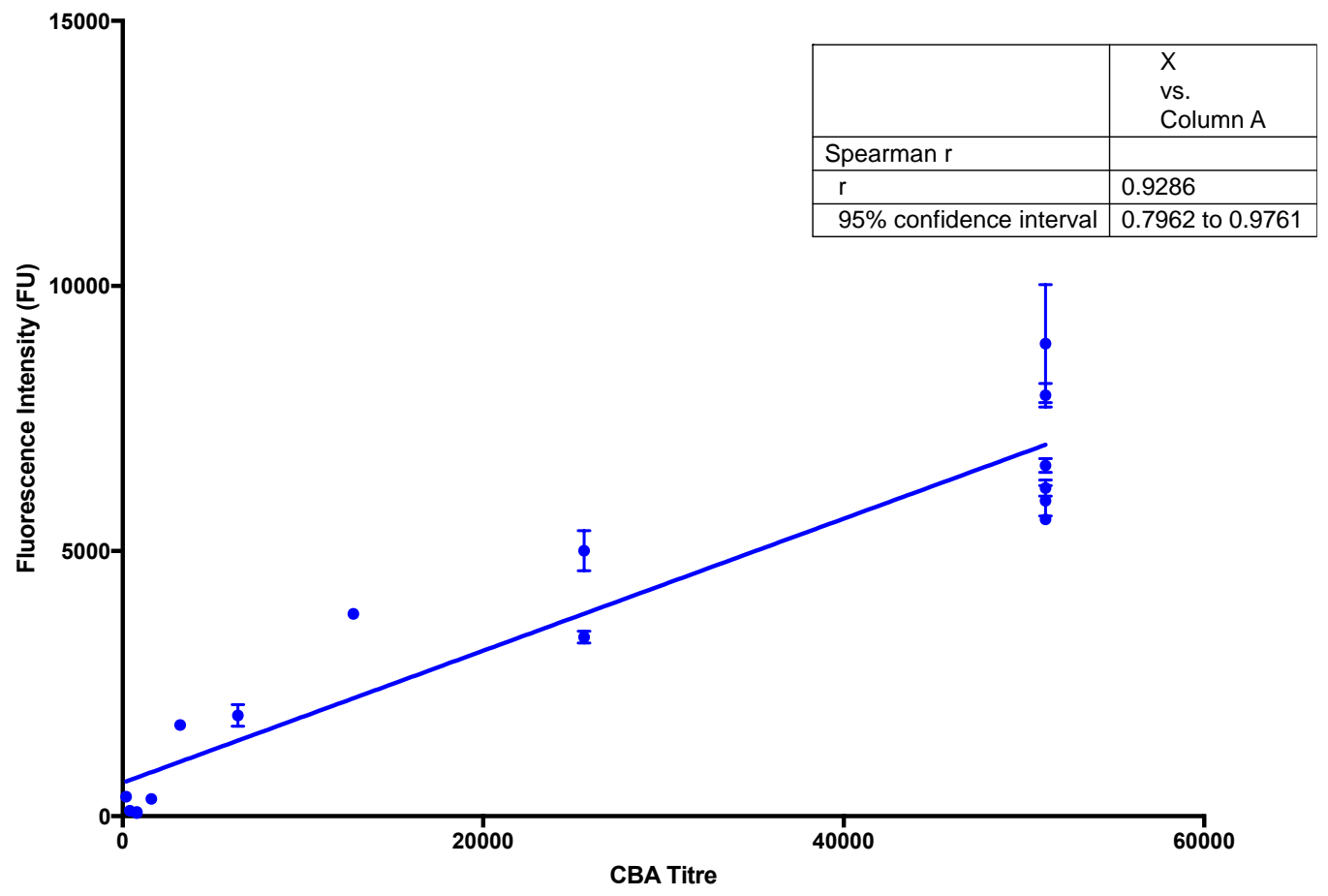

